\title{
Small Satellite Operations Model to Assess Data and Energy Flows
}

\author{
Sara Spangelo * and James Cutler ${ }^{\dagger}$
}

\begin{abstract}
This paper introduces a mathematical model describing the energy and information systems for small satellite mission architectures. We introduce an analytic satellite model, represented by realistic constraints on the available energy, link characteristics, and data and energy storage capacity on-board a small spacecraft. This paper aims to identify the interaction of energy and information flows through the data collection, data processing, and data downlinking modes of operation across the satellite subsystems. Our work augments existing models and tools which quantify network capacity, defined as the information exchange between collections of ground stations and satellites. These models and analyses lay the groundwork for our future goal, to develop optimal scheduling algorithms to maximize the data transfer capacity, where data is transferred from satellites to ground stations within a network. This work develops a toolkit based on analytic modeling to enable network communication analysis using engineering software and a simulation environment. Simulations analyze these exchanges using a representative CubeSat mission with a dedicated science objective. In this initial assessment we aim to quantify the sensitivity of the model to the parameter inputs, and how the satellite design and operation decisions influence the ability to transfer data for small satellites.
\end{abstract}

\section{Introduction}

Current small satellite developers face the challenges of complex communication systems and the restrictions of on-board satellite power and data constraints. There has been a recent trend towards small satellites due to their attractive cost and development time scales. Small satellites are classified as being under $500 \mathrm{~kg}$, and this paper emphasizes CubeSat nanosatellites, which are 1-10 kg in mass. The missions and capabilities of small satellites, including the downlink of science and telemetry data, are limited by monolithic designs, reliability issues, and high mission costs. ${ }^{1}$ We are motivated to enable scientists to perform missions that have conventionally been impossible with single small nanosatellites. Our vision is to support multiple satellites performing science missions in concert, collecting, communicating, and downlinking data. For example, consider the QB50 Project, a nanosatellite science network consisting of 50 international CubeSats collecting multi-point, in-situ measurements of the atmosphere in the lower thermosphere and performing re-entry research. ${ }^{2}$ Current spacecraft and ground station infrastructure limits the capabilities of this type of mission, due to the restrictions in transferring large amounts of science data to the Earth. We aim to overcome this constraint with a dynamic federated ground station network (FGSN) ${ }^{3}$ that enhances coverage to satellites and enables worldwide connectivity. The goal is to maximize the data transfer capacity, defined as the information exchanged between collections of ground stations and satellites which create federated networks.

In this paper, we develop the foundation for optimization algorithms which will distribute excess ground station capacity to satellite users through intelligent deployment coordination and flexible scheduling. This work focuses on modeling the satellites within a communication network, to augment the work in Reference 3, where models and tools for assessing network capacity from the ground station perspective are presented. We develop a mathematical satellite model for the exchange of energy and data, and study the relationship between these two resources for small satellite mission architectures. The analytic satellite model introduced is represented by realistic constraints on the on-orbit available energy, link characteristics, and data and energy storage capacity. The assessment aims to identify the interaction of energy and information flows through the data collection, data processing, and data downlinking operational modes across the satellite subsystems. Through numeric simulations we analyze these exchanges using a representative small satellite with a dedicated science mission.

\footnotetext{
*Ph.D. Candidate, Aerospace Engineering, University of Michigan, 1320 Beal Ave, Ann Arbor, MI 48109.
}

${ }^{\dagger}$ Assistant Professor, Aerospace Engineering, University of Michigan, 1320 Beal Ave, Ann Arbor, MI 48109. 


\section{A. Existing Literature}

The small satellite paradigm encompassed by the slogan "Faster, Better, Smaller, Cheaper" emerged in the late 1980s, enabling innovative space mission architectures with a new class of space applications. Early satellite models focused on the financial trade-offs in small spacecraft design. Mosher describes the Small Satellite Cost and Design Model (SSCM and SSDM) developed by the Aerospace Corporation to achieve design-to-cost goals for spacecraft built with commercially off-the-shelf components and minimize non-recurring development costs. ${ }^{4}$ This tool includes high level modeling of the relationships between the satellite subsystems, where distributions of the financial costs associated with the physical, functional, and developmental characteristics of highly constrained small spacecraft design are studied.

In the literature, there are several models which have been developed for spacecraft operations, which often consider one or a subset of the subsystems such as data handling or the electrical power system to varying degrees of fidelity. McFadden et al. introduce a data handling and operations model for the Fast Auroral Snapshot Explorer (FAST) satellite. ${ }^{5}$ Daily science and real-time commands are balanced to optimize high daily science collection. The power cycling required to maintain a positive energy balance is also studied in their work.

Spacecraft dynamics have been modeled independently of the spacecraft subsystems for a single mission application in References 6, 7, and 8, and a spacecraft propulsion system is modeled in Reference 9. Commercially available simulation environments are used by many authors to model spacecraft dynamics and/or communication systems. Reference 10 emphasizes control system design and model controlled vehicles using MATLAB. A radio-frequency modeling tool called the Communications System Taxonomy (CommTax) interfaced with Analytical Graphics, Inc. (AGI) enables engineers to model communication and the interoperation of multiple nodes in Reference 11.

Kuwahara et al. focused on the operational modes across multiple satellite subsystems in the hierarchical system architecture for the "Flying Laptop" satellite with a field programmable gate array. ${ }^{12}$ This system includes attitude control, ground communication, power management, and on-board data handling using a state-machine diagram and implemented using Math Works Simulink/Stateflow Toolbox. A modular, reconfigurable application framework, the Automated Planning/Scheduling Environment (ASPEN), is designed to support a range of planning and scheduling applications for spacecraft and large aircraft and rapid development of automated scheduling systems for NASA. ${ }^{13}$ This framework contains an expansive constraint modeling language for users to define operability and resource constraints and a graphical interface for visualizing plans and schedules.

The existing models and simulation tools suffer from a fragmentation across multiple disciplines and there is a definite need for multidisciplinary model and simulation tools. ${ }^{14}$ The high fidelity models developed in the literature are generally for a single mission, and often consider only a single subsystem or subset of the subsystems, lacking the flexibility to be applied to an extensive array of missions where the subsystems may have unique and diverse interactions. The existing models also lack a clear analytical structure which may provide insight into the underlying system trade-offs. Furthermore, previous work has not focused on modeling small satellites subject to restrictive and dynamic satellite mission objectives and constraints. Our work aims to first develop high fidelity mathematical models and then based on this foundation, create simulation tools which enable us to perform design, analysis, operational planning, and optimization on a large variety of small spacecraft architectures.

\section{B. Unique Contributions}

Our work focuses on developing general analytic models for small spacecraft operations which can be used to describe a broad spectrum of mission capabilities and constraints. We apply these models to a simulation environment and focus on the coupling and trade-offs between the subsystems, in particular the power and communication systems. We develop analytic representations for the trade-offs between the system resources observed through simulation to aid in future design decisions and operations optimization. Our larger goal is to develop robust, real-time optimization algorithms for multi-satellite missions and federated ground station networks. Our initial unique contributions to this objective are listed below.

1. Develop general analytical models for small satellite operations as a function of mission requirements and constraints.

2. Develop a numeric simulation toolkit to implement these models on small satellite systems.

3. Use our model and tools to study the interaction between data and energy flows for different small satellite mission architectures. We study the sensitivity of network capacity to satellite model constraints. 


\section{Network Capacity Model}

The mathematical model developed in this paper assesses network capacity, driven by the needs for data exchange and satellite to ground station communication link availability. A communication network is a collection of links and nodes which connect to enable the exchange of information. The nodes are data sources and sinks that exchange information over links.

\section{A. Capacity Definition}

The total capacity $C_{N}$ of a given network $N$ is the summed capacity over the set of satellites $I$ and ground stations $J$. In prior work (Reference 3), we assessed the capacity from the ground station perspective and studied the capacity of individual ground station nodes $\left(C_{j}\right)$. In our current spacecraft modeling work, we are interested in the capacity from the satellite perspective, and therefore represent the capacity of the network as,

$$
C_{N}=\sum_{i \in I} C_{i}
$$

where $C_{i}$ is the capacity of a single satellite $i . C_{i}$ is computed by summing the integrated data transfer rates between each ground station $j$ and the space node $i$ throughout the full time period of interest, $t=\left[t_{o}, T\right]$.

$$
C_{i}=\sum_{j \in J} \int_{t_{o}}^{T} a_{i j}(t) r_{i j}(t) l_{i j}(t) \eta_{i j}(t) d t,
$$

In Equation 2, $a_{i j}(t)$ represents the availability of a link (the existence of a line-of-sight) and the data transfer rate is $r_{i j}(t)$. The establishment of a communication link, driven by the ground station schedule and satellite operational modes, is represented by $l_{i j}(t)$, and the efficiency of the satellite and ground station is $\eta_{i j}(t)$. The influence of the ground station on these four capacity parameters are described in detail in Reference 3. In the following discussion we focus on modeling these parameters from the satellite perspective.

\section{B. Satellite Model}

To improve the accuracy of our network capacity representation, we develop a model for the communication parameters from the perspective of a small satellite. Our prior network modeling assumed that the space node can always maintain an ideal communication link when a line-of-sight exists to a ground station. ${ }^{3}$ With the introduction of realistic spacecraft constraints, the fidelity of the model increases, and total network capacity will generally decrease. Throughout the remainder of the paper, we model a single spacecraft; therefore the subscripts on data and power rates from section are not used for simplicity. The model we introduce may easily be extended to consider multiple spacecraft within the network. We now introduce three important elements in the network model: the energy, link budget, and data storage capacity constraints.

We first consider the crucial energetic constraints of the mission and satellite. The goal is to ensure there is sufficient energy to support the energy-consuming operational modes of data collection, processing, and downlinking. The mission dynamics and objectives govern the spacecraft location and operational modes. We must consider when the satellite is in view of the sun to collect solar energy, the available access times to experimental zones, and when there may be ground stations available to downlink data. The energy available to perform tasks is constrained by the available solar power, the power requirements of the other subsystems, and the battery dynamics. The spacecraft energy budget must satisfy,

$$
\begin{gathered}
E(t) \geq E_{\text {min }} \quad \forall t \in\left[t_{o}, T\right], \\
E(t)=E_{\text {in }}(t)-E_{\text {out }}(t)=\eta_{s} \int_{t_{0}}^{t} P_{\text {sol }} d t+E_{0}-\eta_{x} \int_{t_{0}}^{t}\left(P_{\text {op }}+P_{p r}(t)+P_{d l}(t)\right) d t \quad \forall t \in\left[t_{o}, T\right],
\end{gathered}
$$

where $E_{\min }$ is the minimum allowable battery level. $P_{s o l}$ is the power collected from the solar cells and $E_{0}$ is the available energy stored in the battery at time $t_{o}$. The power consuming operations we consider are nominal spacecraft operations, data processing, and data downloading, $P_{o p}, P_{p r}(t)$, and $P_{d l}(t)$, respectively. The total operational power requirement, $P_{o p}$, includes all housekeeping and payload operations unrelated to communications, including the attitude determination and control system, payload operations, position and time system, data collection, and the 
flight computer power needs. The dynamic power required to process the data, $P_{p r}(t)$, is mission specific and related to other spacecraft operations such as data collection and downlinking. The power necessary to downlink the data, $P_{d l}(t)$, may take on different values when the satellite closes the link with ground stations with diverse communication capabilities. The power collected and extracted from the power system are scaled by the efficiency terms $\eta_{s}$ and $\eta_{x}$, respectively. These terms characterize losses in storing collected energy and extracting energy to make it available for use. The efficiency terms are a function of the battery charging and discharging characteristics, power system efficiencies, and related to the expected frequency of the charging cycle.

The energy collected by the spacecraft is a function of the intensity of the incident light from the sun and duration of time the satellite surfaces are exposed to sunlight. The instantaneous power collected from the sun is dependent on the solar cell efficiency $\eta_{s o l}$, the solar spectral density $P_{s d}$, the total solar cell surface area $S$, and the solar incidence angle of each surface, $\kappa$,

$$
\begin{aligned}
& P_{\text {sol }}=\eta_{s o l} P_{s d} S \cos \kappa \quad \text { if } \cos (\gamma) \geq 0, \\
& =0 \quad \text { if } \cos (\gamma)<0 .
\end{aligned}
$$

The angle between the line-of-sight from the spacecraft to the sun and the vector normal to each satellite surface is the solar incidence angle, and related to the position and attitude of the vehicle. ${ }^{15}$

The energy stored on-board the satellite at any time instant is limited by $E_{b a t}$, the battery capacity.

$$
E(t) \leq E_{\text {bat }} \forall t \in\left[t_{o}, T\right] .
$$

When the battery is fully charged, the accumulation of excess energy may be avoided by preventing further collection by the solar cells or dissipating heat through the resistors. The objective is to maximize the total data throughput, therefore overcharging the battery is avoided by using excess power to support increased data processing, downlinking, or other spacecraft operations.

The link budget establishes the relationship between power and data rate through the minimum required signal-tonoise ratio (SNR) required for communication. ${ }^{16}$ The characteristics of the ground station and satellite communication systems and the minimum required SNR govern both the feasibility of communication and the power required to support the link at a given data rate. We must satisfy the minimum signal-to-noise ratio (SNR) requirement through the link equation, ${ }^{16}$

$$
S N R \leq \frac{P_{d l} G_{t} G_{r} L_{l} L_{s} L_{a}}{k T_{s} r} .
$$

where $G_{t}$ and $G_{r}$ are the gains of the transmit and receive antennas, $L_{l}$ is the transmitter-to-antenna line loss, $L_{s}$ is the space loss (where $L_{s}$ is inversely proportional to $S$, the path length), $L_{a}$ is the transmission path loss, $k$ is the Boltzmann constant, $T_{s}$ is the temperature noise, and $r$ is the data downlink rate. Let $\alpha(t)=\frac{G_{t} G_{r} L_{l} L_{s} L_{a}}{(S N R) k T_{s}}$, where $\alpha(t)$ is dynamic through a communication pass (since the path length $S$ and other communication parameters may vary throughout a pass) and dependent on the ground and space node characteristics. To satisfy the minimum SNR, the maximum rate of data exchange between a satellite and ground station can be expressed,

$$
r(t) \leq \alpha(t) P_{d l}(t) .
$$

The data storage capacity, $D_{\text {cap }}$, limits the total amount of data which can be stored on-board for future download,

$$
\begin{gathered}
D(t) \leq D_{\text {cap }}(t), \\
D(t)=D_{o}+\int_{t_{0}}^{t} r_{c o l}(t) d t-C_{i} \quad \forall t \in\left[t_{o}, T\right],
\end{gathered}
$$

where $D_{o}$ is the data stored in the buffer at time $t_{o}, r_{c o l}(t)$ is the data collection rate, and $C_{i}$ was defined in Section IIA. The length of the available downlink, depending on access times to the available ground stations, and the data rate govern the total amount of data which can be collected, processed, and stored for download later in the mission.

In summary, Equations 4 to 6 establish the available energy to process and downlink data, and coupled with the available access times to ground stations, determine $P_{d l}$. The link equation provides the relationship between the downlinking power and data rate through the minimum required SNR (Equation 8). The total satellite capacity is determined by the data rate and efficiency terms (Equation 2), subject to the constraints in Equations 4-11. Note that the on-board data handling system may be unable to store collected data when the on-board buffer is full, however this 
is not often an active constraint in small spacecraft missions due to the large amounts of data which are collected.We may also impose a minimum data transfer requirement for a given satellite over a certain period, $C_{\min _{i}} \leq C_{i}$, where $C_{m_{i n} i}$ is the required download capacity for satellite $i$. The downlink time period may also be constrained if the mission requires that data be downloaded over a specific ground station or by a certain time.

\section{Satellite Capacity Models}

The network communication problem can be formulated as a multi-scale optimization problem, where models of successively higher fidelity are considered, each considering another layer of realistic constraints. ${ }^{17}$ To study the various models, we make the following assumptions:

- The initial data stored on the spacecraft at time $t_{o}=0$ is zero, $D_{o}=0$. Initial on-board energy values are equivalent to the battery being at its full capacity $E_{0}=E_{b a t}$.

- We neglect the dynamics of the power requirements and assume $P_{o p}$ is constant.

- The energy required to collect information is small relative to the other energy sinks (data processing and downlinking).

- $D_{c a p}$ is large and is not imposed as a constraint as in general it is not an active constraint, modeled to represent current data storage systems.

- We consider only data downlinking, and neglect the often less constrained data uplinking.

We next introduce the network models and describe how the additive constraints within each model influence the network capacity problem.

\section{A. Maximum Model}

The maximum model assumes a satellite is always in view of the ground station and available for communication, $a(t)=1 \forall t$. Within the framework of this model, we assume on-board energy is not constrained, such that we have the ability to download constantly from the space to ground node. This analysis is important in characterizing the maximum capacity of the communication system, providing a baseline for ideal constant communication. We assess the amount of data which may be downloaded as a function of the radio design, power system, and on-board data handling and storage systems. The power to download, $P_{d l}$, is constrained by the available power of the spacecraft bus, however we assume unlimited energy is available to perform any tasks.

We make the assumption of constant path length $S$ between the ground and space nodes, such that $\alpha(t)$ is constant throughout each pass, which is representative of high orbits. In this model we assume a constant rate of data transmission for a fixed download power, and the total capacity from Equation 2 simplifies to $C_{i}=r T$. We have made the assumption of unlimited storage capacity, that is, the on-board storage capabilities do not limit the amount of data we can collect and then store for future downlink.

\section{B. Topological Model}

Next we study the topological model, where the satellite orbital mechanics ground station location are considered. In this model we include the availability and feasible rates of data exchange for the dynamic problem, $a(t)$ and $r(t)$, respectively. We assess the amount of data which may be collected and downloaded for small spacecraft systems, considering the trade-off between the available solar power and required power to achieve the minimum signal strength required for downlinking throughout each ground station pass. We assume that the stored on-board energy never exceeds the battery capacity.

Due to the periodic nature of spacecraft orbits and operations, we model the energy and data exchange characteristics on a cyclic basis of length $T$, assuming all subsystem behavior repeats approximately with each cycle. Note this period may be of arbitrary length ( 1 orbit, 1 day, 1 month, several weeks, etc.), depending on the characteristics of the orbit and mission. Consider the importance of perpetuity in the energy and data dynamics of spacecraft operations. After each period, the spacecraft must restore (through solar collection) as much energy as was consumed in operations, processing, and downlinking,

$$
\eta_{s} \int_{0}^{t} P_{s o l}(t) d t \geq \eta_{x} \int_{0}^{t}\left(P_{o p}+P_{p r}(t)+P_{d l}(t)\right) d t \forall t \in[0, T] .
$$


$P_{d l}(t)>0$ only when downlinking occurs, which implies a line-of-sight is available between the communication nodes, $a(t)=1$, and the data rate is nonzero, $r(t)>0 . P_{d l}(t)=0$ at all other instances. Throughout the remainder of the paper we will refer to this expression as Constraint 12 as it is a necessary constraint to satisfy the perpetuity requirement of small satellite missions. We must also ensure that the on-board stored energy never exceeds the battery capacity throughout the mission, Equation 7, nor falls below the acceptable battery discharge level, Equation 3. Satisfying these energy balance constraints is crucial in guaranteeing perpetuity of the mission. Constraint 12 controls if and for what duration data may be downlinked, and also determines the feasible data rates in order to satisfy the dynamic link relationship. The capacity equation is now a function of the availability of a line of sight, $a(t)$, the feasible data transfer rate, $r(t)$, and must satisfy the energy constraints of the mission. For the topological model, Equation 2 reduces to,

$$
C_{t o p}=\int_{0}^{T} a(t) r(t) d t
$$

\section{Actualized Model}

The final model of highest fidelity considers all of the scheduling constraints and inefficiencies of the communication network. We consider the operational constraints of the satellite, $l_{j}(t)$, and the scheduling constraints of the ground station, $l_{j}(t)$, to populate the link parameter, $l_{i j}(t)=l_{i}(t) l_{j}(t)$. The satellite operational modes and on-board computing and power resources must be considered when populating $l_{i}(t)$. In particular, we must include when the spacecraft is collecting, processing, and compressing data according to the satellite mission requirements. Due to these scheduling limitations, the satellite may only be able to downlink during certain periods of operation, reducing the available downlink windows. Due to the limitations in slewing and tuning conventional small satellite ground communication systems, we impose the realistic constraint that each ground station is only able to maintain a link with a single satellite at any instant in time. The scenario where multiple spacecraft are in view of a single ground station is characteristic of multiple spacecraft deployments shortly after launch. The result is that the total usable network capacity decreases when we consider the overlap times of the orbiting satellites. The major influence of the added scheduling constraints is a reduction in feasible downlinking time. With reduced windows of communication within the framework of this model, it may be more energy and time efficient to transmit at a higher data rate and the resultant higher power (to meet the minimum SNR) for a shorter duration.

Less capacity is expected relative to the capacity in the proceeding models due to a reduction in solution space with the added scheduling and efficiency constraints. The capacity in the actualized model is,

$$
C_{a c t}=\int_{o}^{T} a(t) r(t) l(t) \eta(t) d t .
$$

\section{Simulation Environment}

Currently available software tools do not allow us to monitor the dynamic exchange of energy and data with the level of fidelity we are interested in analyzing. Therefore, built on our models, we develop a simulation tool to study the exchange of system resources for small satellite operations. Our initial toolkit models the system level flow of energy and data throughout a mission subject to realistic operational constraints.

The typical operational flows of energy and data on-board a small spacecraft are now briefly described. The energy and data sinks and sources are unique to the mission and satellite capabilities. Power is collected at some nominal rate dependent on the position and attitude of the spacecraft in orbit. We use a solar model which uses the vector to the sun relative to the center of the spacecraft and the instantaneous location of each body face (a product of the rotations and initial conditions of the satellite) to compute the solar incidence angle of each solar panel relative to the sun. We assume that there is no shadowing or interference from other obstructions in space or on the Earth, ideal solar cell circuitry, and that the solar cell operating temperature is constant and ideal. Note that in on-orbit conditions, the temperature will vary as a function of the time history of the cell exposure. Future work will address the solar cell efficiency as a function of cell temperature. The collected power immediately supports on-going operations, or if the available power exceeds the instantaneous system power needs, is stored as excess energy in the on-board battery. The power consumption and the type of mission, including orbit and operational modes of the spacecraft, are interdependent. A spacecraft with a dedicated mission will have deterministic energy dynamics, and the flow of energy in and out of

the system is easily modeled, based on the data collection, processing, and downlinking opportunities and needs. In the case of opportunistic mission operations, the spacecraft data collection opportunities are stochastic in nature, and 
influence downstream data and energy flows in non-deterministic ways. These systems may require stochastic models and become increasingly complex due to the dynamic coupling of the satellite subsystems and operations.

\section{A. Simulation Architecture}

The simulation environment is shown schematically in Figure 1, where the elements of satellite subsystems and operations are identified. The first stage is an identification of the mission, satellite constraints, and ground station characteristics. This combined information is input into Satellite Tool Kit (STK) or in-house developed scripts based on analytic models written in MATLAB to determine the availability of line-of-sights between the satellite and targets of interest. We assess the access times of a given satellite relative to experimental zones, other satellites, and ground nodes as the collection of science or surveillance data and communication require visibility to these geographic locations. Two Line Elements (TLEs) from launched spacecraft or the predicted orbits of future deployments can be used with STK models to generate this information. Our high fidelity orbital propagators consider the dynamics of the spacecraft motion, control and disturbance forces and moments influencing spacecraft motion and attitude, elevation requirements of the ground station, and pointing capabilities of the spacecraft. Our numeric tools are based on analytic expressions which enable us to examine the trends in available access times and their relationship to the space and ground node locations and dynamics. We are able to study short period trends such as the variation in duration of pass between orbital periods as well as the long term variations due to the Earth's oblateness, aerodynamic drag, and separation effects between satellites deployed in the same satellite cluster. In parallel to assessing the availability of data collection and downlinking opportunities, we assess the link budget between communication nodes within the network.

We use the outputs from this assessment in the next phase of the simulation environment to compute the network communication capacity by evaluating the data and energy dynamics throughout the mission. Here we impose the realistic constraints of the network and satellite, in particular the scheduling constraints and upper and lower bounds of the on-board capacity to store energy and data. We integrate power collected by the solar cells and consumed in nominal operations, data collection, processing, and downlinking continuously throughout the mission. In constructing the energy and data time histories, we use short-term predictive methods to assess whether the constraints will be violated, and therefore make decisions on the ability to perform energy consuming tasks such as data processing and downlinking. For example, if a future download will deplete the battery below the acceptable level, we chose to downlink only until this minimum level is reached. We recognize that the optimal solution is by no means reached using this heuristic approach, as we essentially downlink at every possibly opportunity, neglecting future (potentially more time or energy efficient) opportunities to downlink. We are currently working at formulating and solving this optimization problem with the objective function to maximize total data transfer subject to the mission constraints.

\section{B. Mission Scenarios}

It is important that our models and tools can easily be applied to a range of mission scenarios given the diversity of current and future missions. To aid in building representative models for simulation and optimization, we classify spacecraft according to their mission requirements, in particular according to how and when data is collected, processed, and downlinked. The on-board energy dynamics will also be characteristic of the mission type, due to the inherent link between data and energy flows. The models and tools developed in Sections II and IVA are general enough to capture the elements and complexity of any of the operational scenarios we introduce. For example, consider three modes of satellite operations:

1. Focused data collection and downlink mode: Data collection is deterministic and occurs when the satellite passes over given targets at known times, and the downlinking schedule is pre-determined. An example is a science mission such as the Radio Aurora eXplorer (RAX) ${ }^{18}$ designed to collect science data over known experimental zones and downlink to dedicated ground stations.

2. Opportunistic mode: In this mode, data collection occurs when triggered by an event (which may be stochastic), and the satellite must process/downlink according to satellite constraints and mission objectives. The QB50 Project $^{2}$ is an example where multi-point, in-situ measurements will be collected as triggered by atmospheric behavior. Missions where immediate images are required of certain Earth or space locations in response to ongoing activity are also opportunistic in nature.

3. 24/7 mode: The satellite is collecting data or performing testing on a continual basis. This mode must be designed to handle dynamics in available incoming energy and data with intelligent power and data handling 


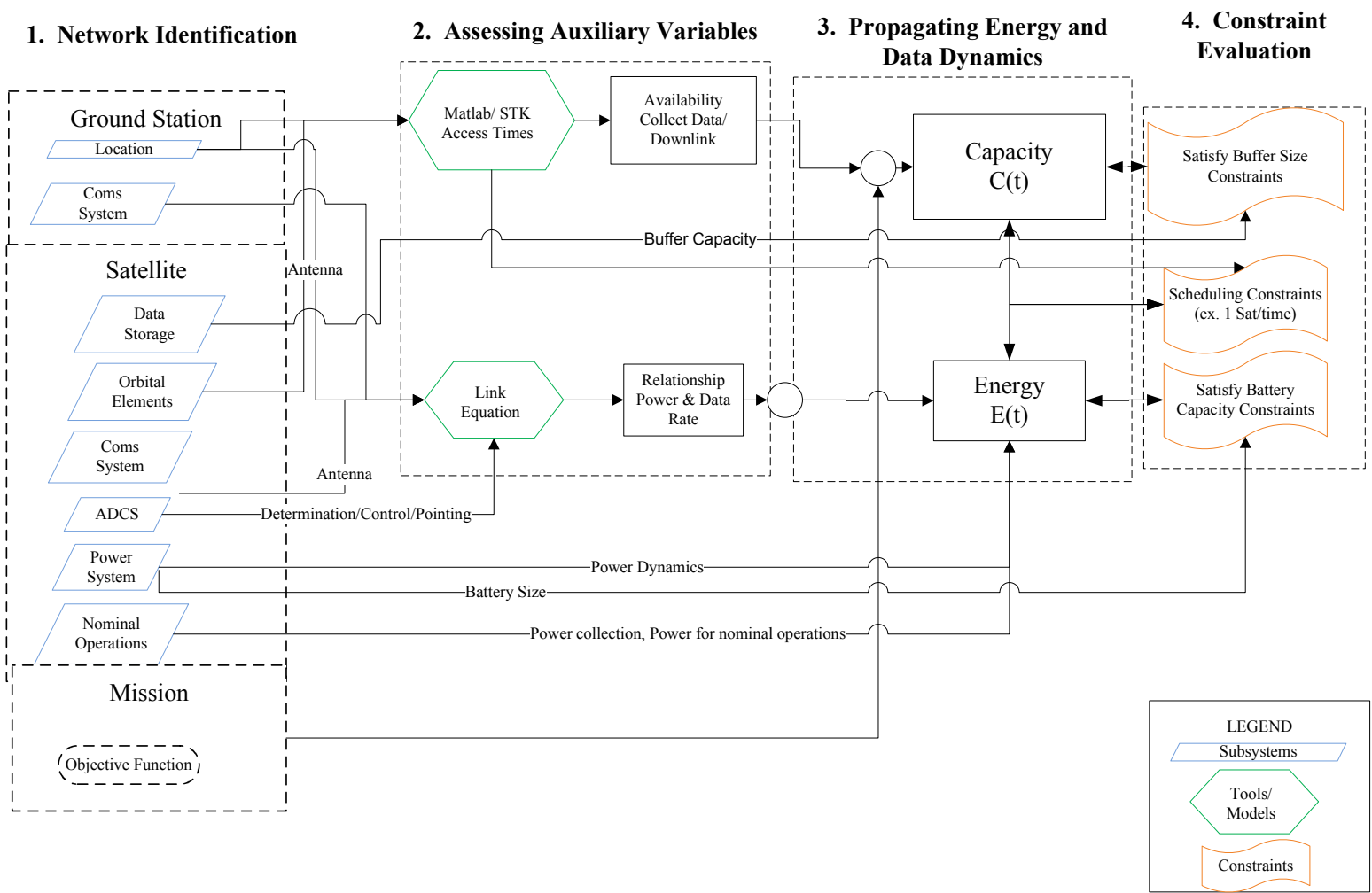

Figure 1. Satellite spacecraft operations simulation architecture. 
schemes. For example, the Disaster Monitoring Constellation operated by the Algerian, Nigerian, Turkish, British, and Chinese governments provides constant and reliable global coverage. ${ }^{19}$ This network provides images for emergency disaster relief in less than a day in response to requests.

In some cases, the focused or opportunistic modes will extend to the $24 / 7$ coverage mode. A single mission may be designed to have multiple modes of operation, where during dedicated times is it performing required mission tasks and between these scheduled intervals may enter opportunistic or constant modes of operation. The first mode is deterministic; mission designers can easily predict expected data rates, data processing requirements, and network capacity prior to the satellite deployment. Stochastic models are required for the second and third modes of operation, and bounds on the amount of data collected are extremely complex to predict apriori.

\section{Example Mission Scenario}

As an example, consider a representative mission which operates in the focused science data collection and downlink mode. The spacecraft we model is the first National Science Foundation (NSF) funded CubeSat, the Radio Aurora eXplorer (RAX) ${ }^{18}$ RAX a $3 \mathrm{U}$ nanosatellite, approximately $10 \mathrm{~cm}$ x $10 \mathrm{~cm}$ x $30 \mathrm{~cm}$ in size and weighing less than $3 \mathrm{~kg}$. The spacecraft is designed to study space weather in the ionosphere. RAX collects science data from field-aligned plasma irregularities (FAIs) from incoherent scattered radar stations (ISR). The primary station is located in Poker Flats, Alaska. The science collection will occur several times a day when the spacecraft passes over the 2,400 km diameter experimental zone centered at the ISR in Alaska. RAX then process the data and proceeds to downlink the science and telemetry data to two ground stations located at the University of Michigan in Ann Arbor, Michigan, and at SRI International in California. The ground station at SRI International has sufficient gain at $2.4 \mathrm{GHz}$ to operate at the maximum radio data rate of $115.2 \mathrm{kbps}$. The Ultra High Frequency (UHF) antenna at the University of Michigan communicates at approximately $430-440 \mathrm{MHz}$ and expected data rates are $9600 \mathrm{bps}$.

We model the representative RAX science mission using our simulation environment over a twenty-four hour period. Figures 3 and 2 show the data and energy time-histories of the mission generated with our simulator. Figure 3a) shows the level of stored energy on-board the satellite as a result of the power dynamics (Figure 2a)), while Figure 3b) captures the flow of science data collected, processed, and downlinked throughout the satellite mission. This mission collects science data when it has a feasible line-of-sight to the experimental zone, and then proceeds to undergo an energy-consuming processing phase. The satellite downlinks compressed data when dedicated ground stations are in view. Energy levels drop significantly when data processing and downlinking occurs, and increase when the satellite is not in eclipse. The large increments in data buffer size occur when the satellite is within view of experimental zones collecting data, while data downlinking reduces this on-board storage buffer at a rate proportional to the feasible data rates for download. Data is downlinked at two discrete rates $(115.2 \mathrm{kbps}$ and $9600 \mathrm{kbps})$ in accordance with the ground station capabilities, while both UHF and S-band radios require $3.2 \mathrm{~W}$ to downlink. The data rate plot, Figure $2 \mathrm{~b}$ ), is logarithmic to capture the large differences in scales of data collection, data processing, and downlink rates.

Battery sizing is another important consideration in spacecraft design; we aim to minimize the battery size while adhering to mission requirements. The lifetime of a battery is linked directly to the power system operations, and in particular the nominal and maximum depth of battery discharge (how much energy is extracted from the battery on a periodic basis in nominal satellite operations). The battery on-board the RAX spacecraft is sized such that the minimum depth of discharge does not exceed $80 \%$ to preserve its lifetime throughout the expected mission duration. In Figure 3a), immediately following data collection and processing, the energy level is low, although it does not quite reach the minimum allowable battery value $(94 \mathrm{~kJ})$. Following the energy-consuming events (data collection and processing) early in the mission, there are no energy requirements in addition to the nominal operations between approximately 8 and 16 hours in the simulation. During this phase, the energy levels in the battery are restored, and eventually excess power becomes available, where power is available when the vehicle is in view of the sun, however there is no capacity to store it in the on-board battery. The goal is to minimize the power which is unable to be stored in the battery as this makes our mission less energy and time efficient. In our future work we aim to simultaneously design the battery and spacecraft operations to optimize the overall data transfer while meeting mission constraints.

\section{Capacity Assessment}

The objective of this section is to study the sensitivity of the energy and data dynamics to changes in the communication parameters such as the availability, link value, data rates, and communication system inefficiencies. Due to the inherent uncertainty in the inputs to our satellite model, we are interested in quantifying and bounding the variation in the model outputs. This assessment is motivated to aid mission designers and planners in estimating data capacity in 


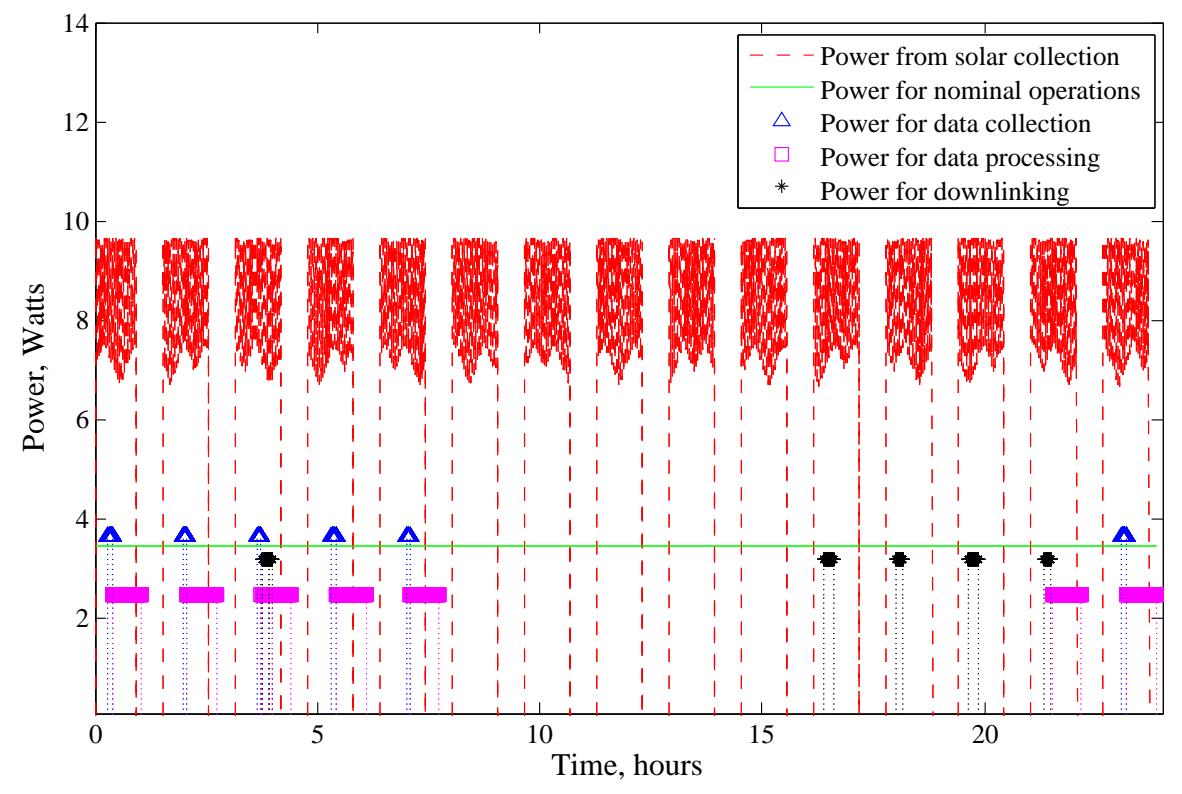

a) Power

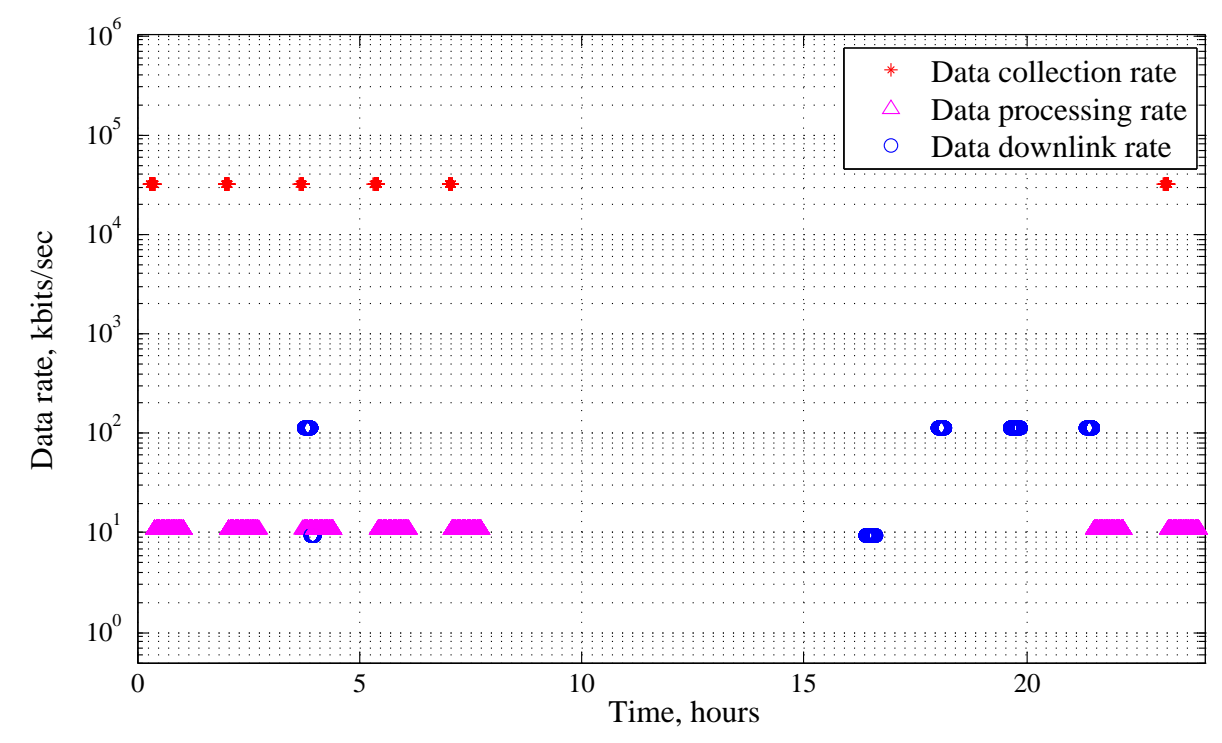

b) Data Rate

Figure 2. Power and energy time histories of RAX Mission for one day. 


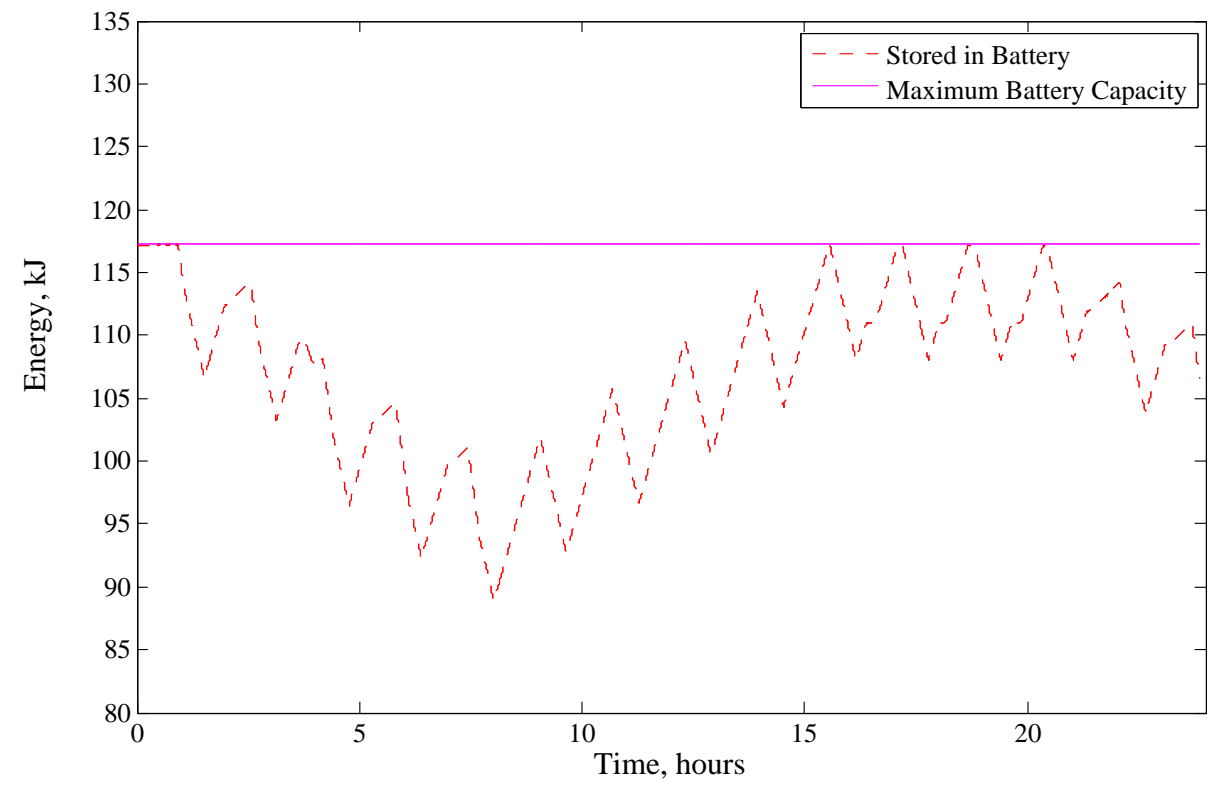

a) Energy

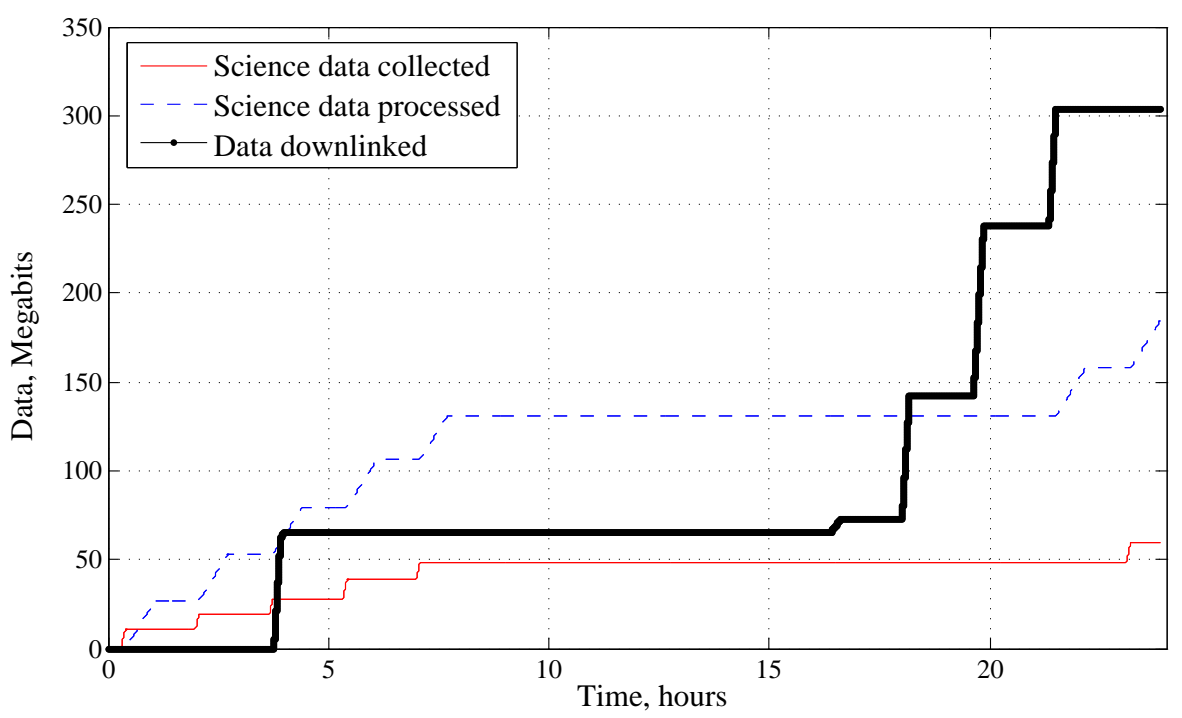

b) Data

Figure 3. Data rates and data time histories of RAX Mission for one day. 
realistic nominal conditions, and understand how variations in the problem parameters influence the capacity. We use the RAX mission as the baseline mission for nominal access times, rates, and constraints. As our goal is generally to maximize the data throughput, we assume we downlink at each opportunity (when a ground station is in view) at the feasible data rate. Future work will further consider optimization of the schedule in order to select the ideal ground stations opportunities and downlink rates.

\section{A. Upper Bound on Capacity}

The upper bound on the capacity for data exchange is useful at the initial design phase and also for operational planning to quantify the maximum potential for data exchange. We have made the assumption of unlimited storage capacity, that is, the on-board storage capabilities do not limit the amount of data we can collect and then store for future downlink. We assume there is a line of sight to an available ground station for the fraction of the time span given by $\eta_{d l}$ and that the spacecraft is able to collect solar energy at an average rate of $P_{\text {sol }}$ for $\eta_{s o l}$ fraction of the time span. We compute the maximum amount of data, $C_{\max }$, which can be transferred during an interval $T$. Constraint 12 is an active equality due to our goal of maximizing the data downlinked. All energy collected will be consumed by communication, nominal operations, or data processing. Assuming we have a perfect ability to store and extract power, $\eta_{s}=1$ and $\eta_{x}=1$, this expression simplifies to,

$$
\eta_{\text {sol }} P_{\text {sol }} T=P_{o p} T+K_{p r} C_{\text {max }}+\eta_{d l} P_{d l} T,
$$

where $K_{p r}$ is the energy per amount of data required for processing (measured in Joules/bit). To maximize the data downlinked, Constraint 9 is active, indicating we are just satisfying the minimum communication requirements. We assume $\alpha$ is constant and independent of $t$, therefore $r=\alpha P_{d l}$ and $r=\frac{C_{\max }}{\eta_{d l} T}$. The total amount of data which may be downlinked is,

$$
C_{\text {max }}=\frac{\left(\eta_{\text {sol }} P_{\text {sol }}-P_{o p}\right) T}{K_{p r}+\frac{1}{\alpha}} .
$$

Equation 16 is based on average values for energy collection and consumption assuming that the on-board energy values never fall below the minimum allowable or exceed the maximum energy capacity of the battery. Assuming there are sufficient downlinking opportunities, note that the maximum communication capacity $C_{\max }$ is independent of $\eta_{d l}$. This is due to the assumption that power and data rate are linearly proportional, indicating that with finite time to downlink, maximizing the data transferred is achieved by simply maximizing downlinking rate while adhering to the energy constraints. This model neglects maximum constraints on $P_{d l}$ and $r$ characteristic of realistic ground and space communication systems.

For the RAX mission, the maximum capacity $C_{\max }$ transmitted over the UHF radio at $9600 \mathrm{bps}$ is $303 \mathrm{Mbits}$ per day using Equation 16 and the mission parameters specified in Appendix A. $C_{\max }$ is 670 Mbits per day over the S-band radios. Note that $P_{d l}=3.2 \mathrm{~W}$ is required to transmit on both radios. The data rates differ by a factor of 12 , while the maximum communication capacity differs by a factor of only 2.2. The upper bounds are not directly related to the downlinking rates due to the nonlinear relationship between $C_{\max }$ and $\alpha$ (where $\alpha$ is inversely proportional to the data rate). Higher data rates for a fixed downlink power result in higher $\alpha$ values, but are combined with the power required to process the data $\left(K_{p r}\right)$, and therefore do not scale directly to the total data capacity. This highlights an important design decision when selecting and operating a satellite radio. There is an analytic connection between the on-board processing and downlinking power requirements and the amount of data transferred, and future multidisciplinary optimization techniques will aim to exploit these relationships. The RAX mission is expected to use both radios, and the single day simulation in the previous section consists of four downlinks on the S-band antenna and two on the UHF antenna. The simulations show that just over $300 \mathrm{Mbits}$ are downlinked once realistic constraints on the energy and data are considered.

\section{B. Parameters Influencing Capacity}

Some of the capacity parameters introduced in Section II are deterministic and predictable, such as the availability of a communication link between a ground station and satellite, while others are stochastic in nature, such as the satellite system failures and ground station downtimes. We next examine each of the communication parameters and discuss their contribution to total network capacity. 


\section{Availability}

General line-of-sight availability information between ground stations and satellites can be attained using standard propagation tools such as Satellite Took Kit (STK). To compliment these tools, we developed analytic tools to assess expected dynamic and average access times as a function of ground station and satellite parameters. We first determine orbital period based on satellite orbital parameters and Earth's rotation rate, and then compute analytically the expected fraction of satellite orbits at a given inclination which will pass over a given ground node latitude. We combine this information with numeric results which provide the average pass duration per orbit to determine estimated access times.

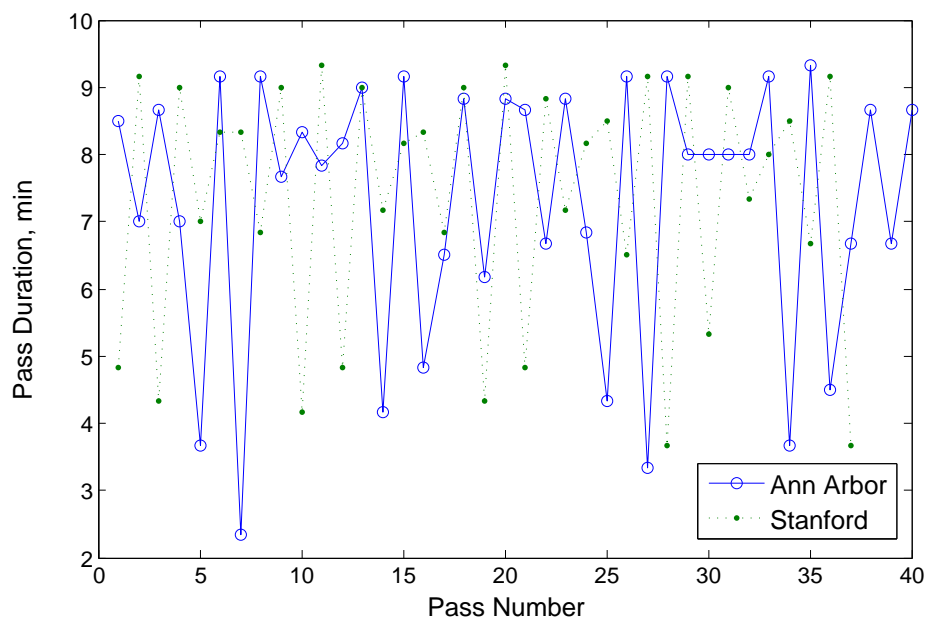

Figure 4. Duration of successive passes for RAX satellite relative to ground stations located at the University of Michigan in Ann Arbor, Michigan and SRI, California with a minimum elevation of $10^{\circ}$.

For example, in Figure 4 we show the characteristic length of sequential passes for the RAX spacecraft relative to its two primary control ground stations in Ann Arbor, MI $\left(42.17^{\circ} \mathrm{N}, 83.45^{\circ} \mathrm{W}\right)$ and SRI, CA $\left(37.25^{\circ} \mathrm{N}, 122.9^{\circ} \mathrm{W}\right)$. Note the difference in the short term frequencies and amplitudes of the pass durations between the two ground station locations, related to the ground station locations. Since satellite orbits are periodic, their trends repeat with predictable patterns due to the interaction of the Earth and satellite orbits, as apparent in Figure 4. This behavior is difficult to describe analytically, and most easily and thoroughly studied using numerical simulation tools, where we can extract the oscillatory behavior.

\section{Data Rate}

The feasible data rates of the satellite may be variable or fixed, depending on the capabilities of the on-board radios. Other parameters related to the data rate such as power consumption and the duration of data transmission, however, may have dynamic behavior. For example, the power required to download will vary slightly throughout each spacecraft pass, dependent on the varying space path losses $L_{s}$ (dependent on the pass length $S$ ) and transmission path loss $L_{a}$, both parameters implicit in the dynamic communication parameter $\alpha(t)$.

The effect of variable data rates on communication performance is studied in Figure 5 for the same scenario introduced in Section IVC. We scale the nominal data rate by the data rate factor, $f_{d r}$. This analysis is of particular interest in selecting optimal data rates to maximize the data exchange across communication networks. The data rate influences the power required to download, and thus has a significant effect on data and energy flows throughout the mission. Since we assume data rate and power are proportional, the required power to transmit scales equivalently to the data rate. We recognize there are other real-life considerations and constraints, such as feasible data rates and power limitations on the satellite and ground station communication systems, but assume for this analysis thesse constraints remain inactive. 


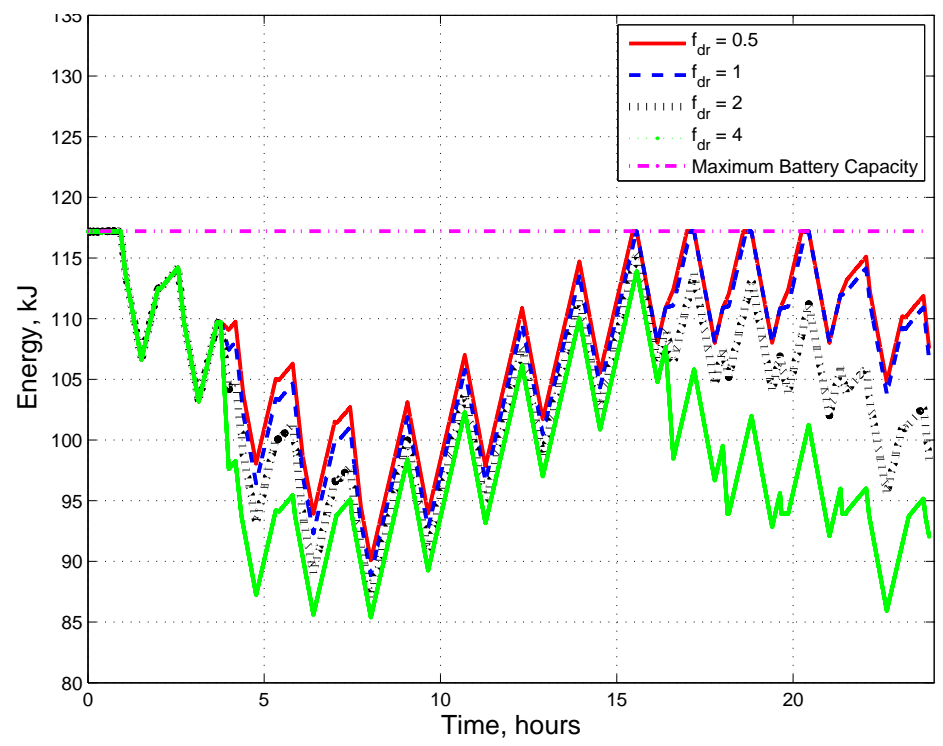

a) Energy stored in on-board battery

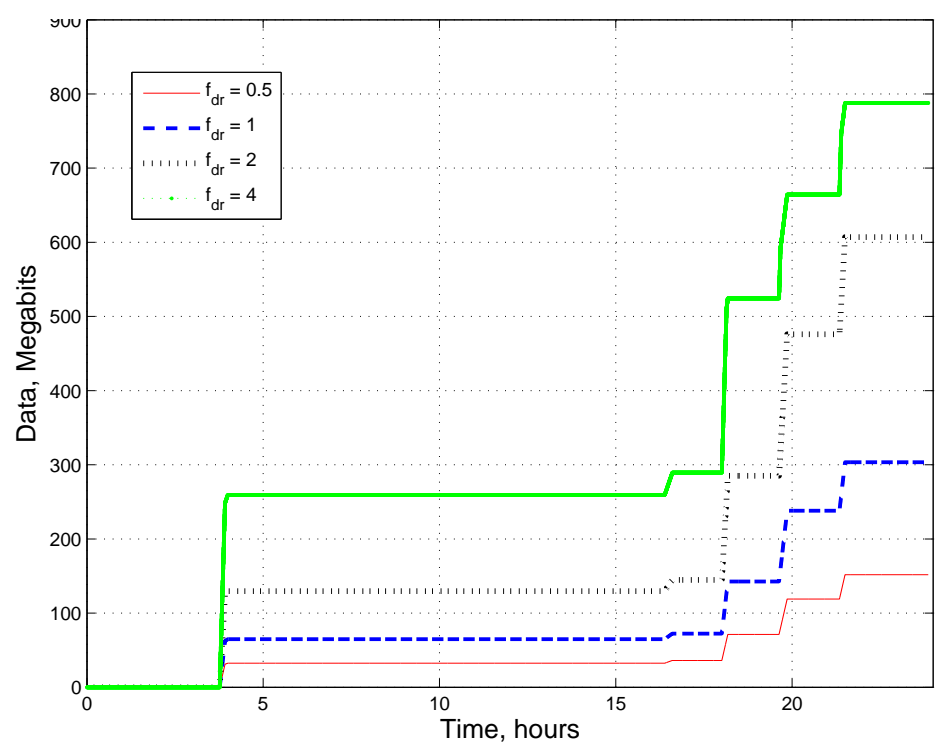

b) Data downlinked

Figure 5. Effect of average communication data rates $\left(f_{d r}\right.$ is the scaling factor) using numeric simulation tools. The simulation is of RAX CubeSat operations for one day with communication parameters $\eta_{a v}=1$ and $l_{a v}=1$ 
The communication capacity after 1 day of operation approximately doubles when we increase the ratio from $f_{d r}=0.5$ to $f_{d r}=1$ (150 and $\left.300 \mathrm{Mbits}\right)$, and then doubles again when $f_{d r}=2$ (600 Mbits). However, this trend does not continue with higher data rate scaling factors. With the higher data rate factor of $f_{d r}=4$, there is only an increase of just over $30 \%$ as compared to the $f_{d r}=2$ case, due to the energy limitations of the problem. The battery is depleted to the minimum allowable discharge level mid-period for the $f_{d r}=4$, preventing further downlinking capabilities. The batter is also discharged close to this level for the $f_{d r}=2$ case. Note the low battery charge after the twenty-four hour period with higher data rates and the repeated and dramatic charging and discharging cycles on the battery. Such extreme and repeated depths of battery discharge are highly undesirable and likely to limit the power system and battery lifetime. Furthermore, the energy at the end of each cycle is critical in studying the potential perpetuity of each scheme. The first two cases, $f_{d r}=0.5$ and 1 have essentially recharged the battery by the end of the cycle, while the $f_{d r}=2$ and $f_{d r}=4$ scenarios have only recharged the battery to $92 \%$ and $84 \%$, respectively.

The most significant trend emerging from these simulations is the crucial tradeoffs between data rates and energy values- higher data rates may not yield larger amounts of data collection. When aiming to maximize the data downlinked with a fixed power generation system, there exists a threshold where there is simply insufficient energy to downlink more data.

\section{Link Feasibility}

In this section we study the impact of the average amount of time an available link is feasible based on the dynamic ground and space node scheduling constraints. The major scheduling constraint faced by the small satellite community is that ground stations are only capable of communicating to a single satellite at a time due to the limitations of slewing and tuning ground antennas to establish communication links. In addition, although single satellites may send data to multiple ground stations simultaneously, in general having multiple ground nodes tuned to a single satellite is a waste of precious ground resources as identical data will be recovered at both ground nodes.

Future science missions will often consist of multiple satellite deployments from the same launch vehicle, such as the QB50 project. As the satellites from a given deployment cluster separate, there will be variable unique access times to ground stations. This will be a function of the initial $\Delta V$ of the launched satellites, aerodynamic drag, orbital seasonal variations due to the Earth's oblateness, and other disturbance forces. In particular, the proximity of the satellites to one another will vary over time, influencing the feasible unique contact times to a given ground station. This effect may be partially modeled based on the known spacecraft dynamics, but will have stochastic elements due to the random nature of the combined effects influencing separation distance.

An example of the separation characteristics of individual satellites and the combined access times of collections of satellites deployed simultaneously is found in Reference 3. For the example where three CubeSats are launched from the same standardized launcher (termed the P-POD), the post-launch total access time of the three satellites was on average $3400 \mathrm{sec} /$ day, only approximately $35 \%$ of the combined individual available access times. This is due to the constraint that a single link may be established between each ground station and satellite simultaneously. We quantify that the average link parameter under these conditions is $l_{a v}=0.35$. In the early stages following deployment, since the spacecraft remain relatively close to one another, their access times overlap, and they are not all able to communicate to the dedicated ground station during the full duration of each pass. After two months, the spacecraft have separated in their orbit due to the reasons discussed above. At this point in time, we've predicted using our simulation environment average access times of approximately $6400 \mathrm{sec} /$ day between the satellite family and the ground station of interest, which is approximately $65 \%$ of the total available unique access times, therefore the average link parameter is $l_{a v}=0.65$.

Models are also needed for the scheduling limitations on-board the satellite, which are related to the mission operations, dynamics of the power collection, and energy storage capabilities. Some of these elements may require stochastic models due to their non-deterministic nature. In addition, since the ground nodes are independently owned and operated, and have the freedom to leave and enter the network, there third party planned or unplanned conflictsof-interest may arise, lowering expected average link values.

\section{Efficiency}

Due to the random nature of failures of both the space and ground nodes, the communication system inefficiencies are modeled probabilistically. From the satellite perspective, we must account for the less than perfect efficiencies of the ADCS, power, and communication systems. Assigning average efficiency values may be insufficient to quantify the potential for total system failures, so stochastic models are required such that the system is designed to be robust to these sudden and potentially devastating shut-downs. Ground station efficiency values as well as satellite and 
total network inefficiencies must be considered. A major contributor to network failures will include transient failures (software glitches), and we can use time to failure and time to recovery metrics to model this uncertainty. An additional consideration to the overall network inefficiencies is the degraded signal strength due to external noise being introduced into the communication system. These effects may occur at random or at deterministic instances (perhaps during regions of high atmospheric disturbances) during the mission, causing a degradation in the SNR and requiring higher power to achieve the desired data rate, or may result in reduced feasible data rates.

We investigate the sensitivity of capacity to changes in the combined satellite and ground station efficiency $\eta_{i j}$. Reference 20 introduced a communication efficiency term $\left(T_{e f f}\right)$ representing the true time a lock is maintained between a space and ground node relative to the available time (when the satellite is in view of the ground station). This work presented experimental data on the efficiency for different maximum satellite elevation passes for LEO satellites communicating to a Vienna ground station in January 2004. The authors found that the ground station efficiency was just below $80 \%$ when the maximum elevation of the pass was $\theta_{\max }=10^{\circ}$, and increased nearly linearly until $T_{\text {eff }}=97 \%$ when $\theta_{\max }=90^{\circ}$. Most of our satellite links are established at a minimum elevation of $10^{\circ}$, therefore we use the range of efficiencies introduced in this study.

\section{Modeling Stochastic Efficiencies}

We assume that the communication link is characterized by discrete efficiency values, where some probabilistic characteristics of the system are known. The efficiency of the communication link is assumed to be in the range $\{0,1\}$, and the set of all discrete efficiency values of a problem is $K$. For example, the link fails $p(\eta=0)$ fraction of the total time, and the ground station performs perfectly $p(\eta=1)$ fraction of the time, and may have probabilities of taking on other discrete efficiencies such that the total probabilities sum to one,

$$
\sum_{k \in K} p\left(\eta_{k}\right)=1
$$

We often know the average communication efficiency, which must satisfy,

$$
\sum_{k \in K} \eta_{k} p\left(\eta_{k}\right)=\eta_{a v}
$$

Once the probability of each discrete efficiency is known, $p\left(\eta_{k}\right) \forall k \in K$, we construct a cumulative distribution function (CDF). The CDF describes the probability that a random efficiency variable with known probability distribution will be found at a value less than $\eta_{k}, p\left(\eta \leq \eta_{k}\right)$. We use the network efficiency probability distribution to populate a set of sample efficiency values. We first generate a random number $\in\{0,1\}$, for example using the rand function in MATLAB. We evaluate the smallest discrete efficiency term $\eta_{k}$ such that $\operatorname{ran}=p\left(X \leq \eta_{k}\right)$ to determine the probabilistic efficiency $\eta$. Depending on the known probability characteristics of a system, we may solve for the other parameters in order to satisfy Constraints 17-18.

\section{Example Stochastic Scenario}

For example, consider a representative scenario where the probability of failure is $5 \%$, and of perfect communication is $5 \%$, that is $p(\eta=0)=p(\eta=1)=0.05$. In addition, assume the known average efficiency is $\eta_{a v}=0.7$. We know the system operates at one other discrete efficiency, of unknown value. The efficiencies are therefore $\eta=\left[\begin{array}{lll}0 & \eta_{2} & 1\end{array}\right]$ and the probabilities are $p=\left[0.05 p\left(\eta_{2}\right)\right.$ 0.05]. Using Constraint 17-18, we have a linear problem with two equations and two unknowns, $\eta_{2}$ and $p\left(\eta_{2}\right)$, which are both constrained to the interval $\{0,1\}$. Solving for the unknowns yields $\eta_{2}=0.72$ and $p\left(\eta_{2}\right)=0.9$. We use this algorithm to populate an efficiency vector for the RAX scenario; where there are 39 time intervals each assigned an efficiency value. The randomly generated probability values and associated efficiency values are shown for each time interval in Figure 6. The probability distribution and CDF are shown in Figure 7, where the probability statistics for this problem are evidently quite close to the probability distribution. In this analysis we assume a link is always feasible between a satellite and ground station $l(t)=1 \forall t \in[0, T]$ over a single day.

Figure 8 shows the effects of random efficiencies with different mean values on data and energy flows throughout a day for the RAX mission. These efficiencies are applied uniquely to the data rate; we assume equivalent energy is consumed in all communication. Problems such as dropped packets, poor visibility, or malfunction of the communication system are the causes for the reduction in transferred data, reducing the capacity of the network by reducing the effective time window for data transfer. The total amount of data collected when the efficiency increases from $\eta_{a v}=0.1$ to $\eta_{a v}=0.5$ increases by a factor of 5.5, an increase from $\eta_{a v}=0.1$ to $\eta_{a v}=0.7$, results in an increase by a factor of nearly 8 , and an increase from $\eta_{a v}=0.1$ to $\eta_{a v}=0.9$, results in an increase by a factor of 8.5. Thus, although higher efficiency values are desired to ensure all possible data is exchanged, the relationship between efficiency and data transferred is not linear. Significant improvements in communication efficiency will not scale directly to improvements in total data transferred. 

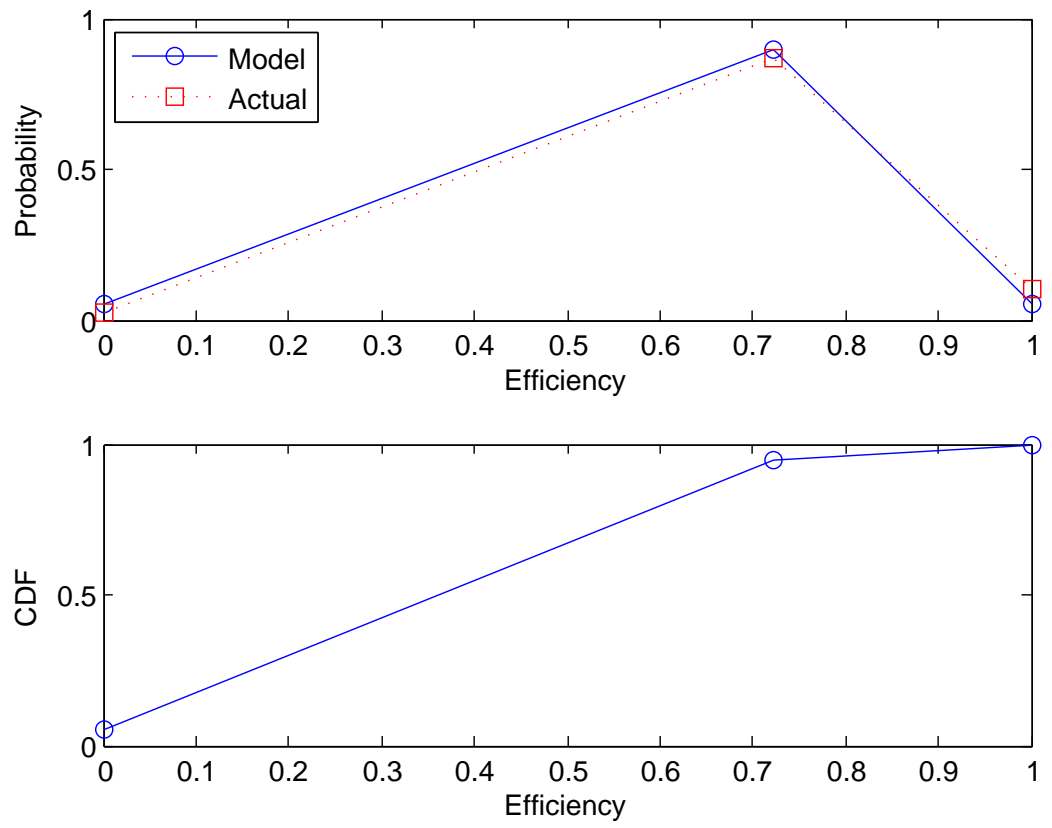

Figure 6. Efficiency cumulative distribution function for the RAX CubeSat operations for one day with communication parameters $f_{d r}=1$ and $l_{a v}=1$.

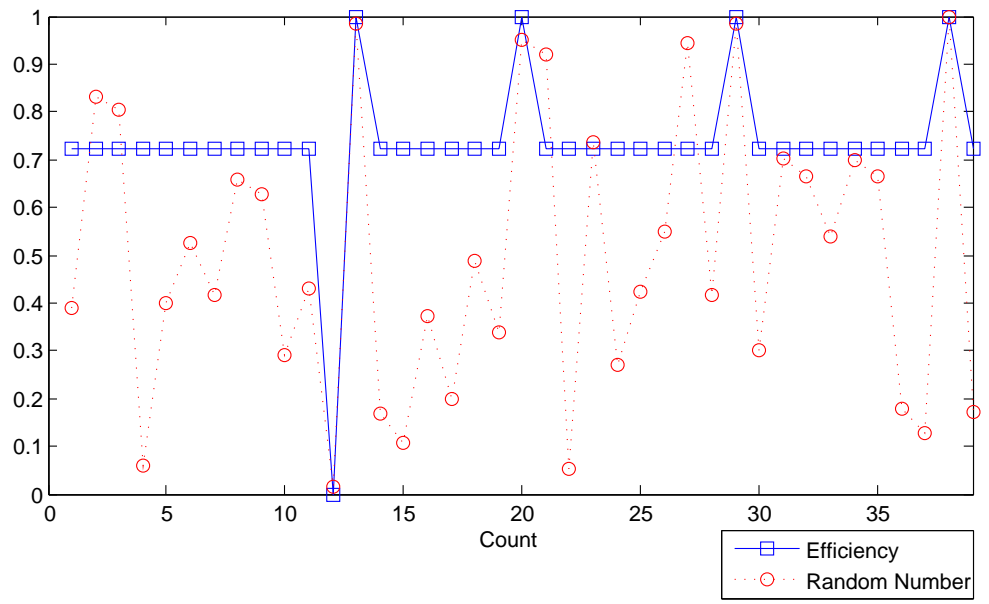

Figure 7. Efficiency distribution for the RAX CubeSat operations for one day with communication parameters $f_{d r}=1$ and $l_{a v}=1$. 


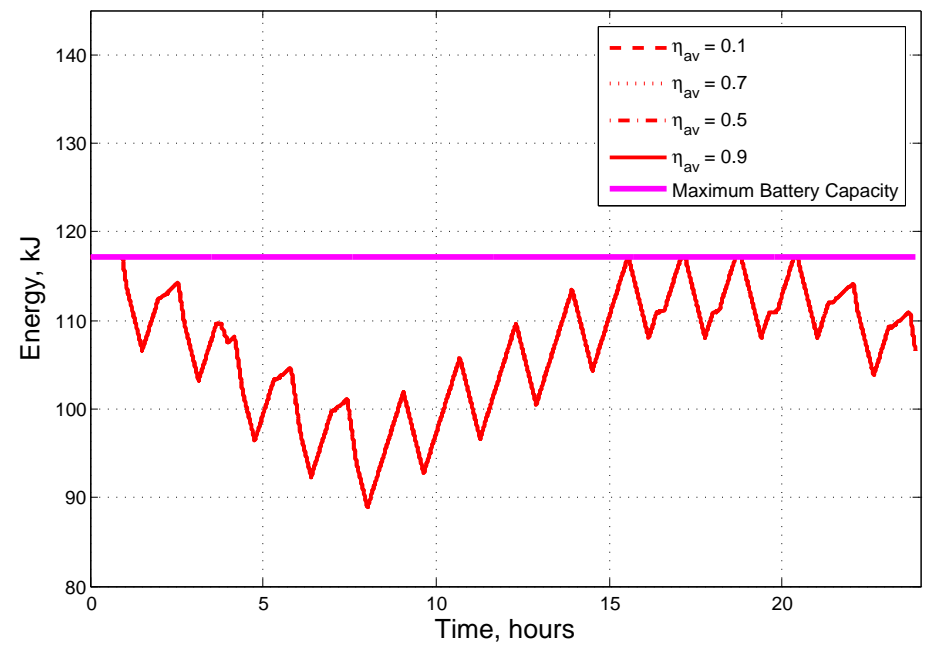

a) Energy stored in on-board battery

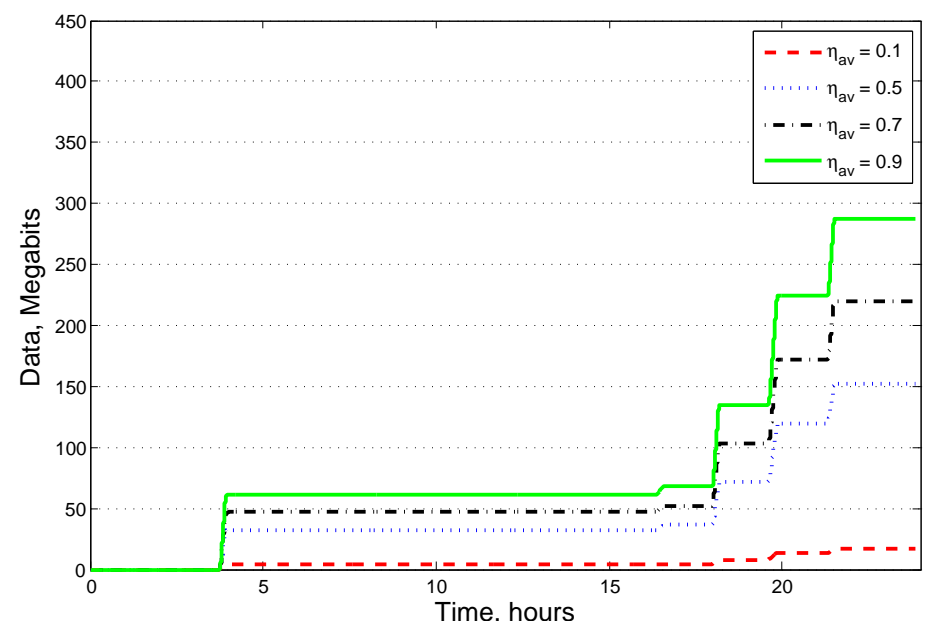

b) Data downlinked

Figure 8. Effect of stochastic efficiency, $\eta(t)$, using numeric simulation tools. The simulation is of RAX CubeSat operations for one day with communication parameters $f_{d r}=1$ and $l_{a v}=1$. 


\section{Conclusions and Future Work}

The models and simulation environment presented in this paper enable us to study the important trade-offs between energy and data using analytic and numeric tools for small satellites. We relate communication trends noted in the simulation work to the analytic formulation of the problem. First, we establish the upper bound on available data transfer given the energy constraints of the mission and spacecraft. Next, we study the parameters influencing capacity, investigating the sensitivity of the data and energy trends to changes in these parameters. Due to the relationship between data rate and power and the limitations on available energy, there is diminishing data transfer return with higher data rates. We discuss the impact of satellite deployment on the schedule parameter $l_{a v}$. Simulations have shown that network efficiency improvements do not scale directly to improvements in communication capacity. These are all important considerations for small satellite mission designers and ground station operators. Note that as we scale the problem to include multiple satellites and ground stations, the interaction of the nodes within the network will cause these trends to evolve, which we plan to address as we further develop and expand our simulation tools for networks of satellites.

The goal of future work is to optimize small satellite communication schedules in a dynamic environment, where both the satellite population and the ground station network are evolving and subject to variable conditions (satellite and ground station failures, change of mission objectives, etc.). In particular, satellites may originate from diverse institutions, and ground stations may not be directly under the control of the team who owns the antenna, forming federations of stations. We aim to optimize the network schedule for the dual goals of balancing station utilization and satisfying satellite communication needs. Satellite scheduling introduces some unique challenges relative to conventional scheduling such as highly constrained vehicles and restricted windows of communication due to the nature of orbiting satellites. These real time tools will facilitate the creation of optimization algorithms for ground station scheduling which can be used for both mission design and tactical network scheduling. Future work will improve existing models and develop real-time algorithms to optimize the schedule for federated ground station networks.

\section{Acknowledgements}

We would like to thank Dr. Andy Klesh, Dr. Eric Gustafson, and the University of Michigan RAX Team for their input and support. We would also like to acknowledge Mark Stakhiv for his support in generating simulation results.

\section{References}

${ }^{1}$ J. Cutler and A. Fox. A Framework for Robust and Flexible Ground Station Networks. In Journal of Aerospace Computing, Information, and Communication, March 2006.

${ }^{2}$ QB50 von Karman Institute for Fuid Dynamics. www. vki.ac. be/QB50/project2 . php, 2009.

${ }^{3}$ S. Spangelo, D. Boone, and J. Cutler. Assessing the Capacity of a Federated Ground Station Network. In IEEE Aerospace Conference Proceedings, March 2009.

${ }^{4}$ T. Mosher, M. Barrera, D. Bearden, and N. Lao. Integration of Small Satellite Cost and Design Models for Improved Conceptual Design-to-Cost. volume 3, pages 97 - 103, New York, NY, USA, 1998.

${ }^{5}$ J.P. McFadden, R.E. Ergun, C.W. Carlson, W. Herrick, J. Loran, J. Vernetti, W. Teitler, K. Bromund, and T. Quinn. Science Operations and Data Handling for the FAST Satellite. Space Science Reviews, 98(1-2):169 - 96, 2001.

${ }^{6}$ R. Schlanbusch, E. Oland, and P.J. Nicklasson. Modeling and Simulation of a Cubesat Using Nonlinear Control in an Elliptic Orbit. pages 632 - 7, Piscataway, NJ, USA, 2009.

${ }^{7}$ S. Rawashdeh, D. Jones, D. Erb, A. Karam, and Jr. Lumpp, J.E. Aerodynamic Attitude Stabilization for a Ram-Facing Cubesat. Advances in the Astronautical Sciences, 133:583 - 95, 2009.

${ }^{8}$ S. Waydo, D. Henry, and M. Campbell. Cubesat Design for LEO-Based Earth Science Missions. volume vol.1, pages 1 435, Piscataway, NJ, USA, 2002.

${ }^{9}$ N.J. Rackemann, H.M. Sanders, and L.D. Van Vliet. Design and Development of a Propulsion System for a CubeSat - Based on Solid Propellant Cool Gas Generator Technology. volume 5, pages 3434 - 3442, Valencia, Spain, 2006.

${ }^{10}$ Jr. A. Bryson. Control of Spacecraft and Aircraft. Princeton University Press, 1994.

${ }^{11}$ D.R. Beering, S. Tseng, J.L. Hayden, A. Corder, Teng Ooi, D. Elwell, H. Grabowski, R. Frederic, J. Franks, R. Fish, A.M. Johnson, and N.E. Gavin. RF Communication Data Model for Satellite Networks. page 7, Piscataway, NJ, USA, 2009.

${ }^{12}$ Toshinori Kuwahara, F. Bohringer, A. Falke, J. Eickhoff, F. Huber, and H.-P. Roser. Operational Design and On-Board Payload Data Processing of the Small Satellite "Flying Laptop" with an FPGA-Based Onboard Computing System. volume 6, pages 3880 - 3887, Glasgow, United kingdom, 2008. 
${ }^{13}$ S. Chien A. Fukunaga, G. Radideau and D. Yan. ASPEN: A Framework for Automated Planning and Scheduling of Spacecraft Control and Operations. Tokyo, Japan,, 1997.

${ }^{14}$ T. Kim P. Bernard, P. Herbert. Theory of Modeling and Simulation. Academic Press, 2 edition, 2000.

${ }^{15}$ S. Spangelo. Solar Collection for Small Satellites in Low Earth Orbit. Tech Memo, Aerospace Engineering, University of Michigan, Ann Arbor, May 2010.

${ }^{16}$ J. Wertz and W. Larson. Space Mission Analysis and Design. Microcosm Press, 3rd edition, 1999.

${ }^{17}$ W.W. Hager, S.-J. Huang, P.M. Pardalos, and O.A. Prokopyev. Multiscale Optimization Methods and Applications. Springer, 2006.

${ }^{18}$ T. Moretto. Cubesat Mission to Investigate Ionospheric Irregularities. In Space Weather: The Journal of Research and Applications, November 2008.

${ }^{19}$ K. Hogie et al. Putting More Internet Nodes in Space. pages 21-23, April/June 2006. Small satellites.

${ }^{20}$ S. Cakaj, W. Keim, and K. Malaric. Communications Duration with Low Earth Orbiting Satellites. pages 85 - 8, Anaheim, CA, USA, 2007. 


\section{A. Spacecraft Simulation Parameters}

Table 1. Simulation parameters for RAX Mission

\begin{tabular}{|c|c|c|}
\hline Mission start date and time & $t_{o}$ & May 28, 2009 0:00:00.00 UTCG \\
\hline Power for data processing & $P_{p r}$ & $2.5 \mathrm{~W}$ \\
\hline Power for nominal operations & $P_{o p}$ & $3.47 \mathrm{~W}$ \\
\hline Power for downloading & $P_{d l}$ & $3.2 \mathrm{~W}$ \\
\hline Maximum battery capacity & $E_{b a t}$ & $117.22 \mathrm{MJoules}$ \\
\hline Rate for data processing & $R_{p r}$ & $11.1 \mathrm{kbits} / \mathrm{sec}$ \\
\hline Rate of data collection & $R_{c o l}$ & $32 \mathrm{Mbits} / \mathrm{sec}$ \\
\hline Energy storage efficiency & $\eta_{s}$ & 0.8 \\
\hline Energy extraction efficiency & $\eta_{x}$ & 0.8 \\
\hline Solar cell efficiency & $\eta_{s o l}$ & 0.24 \\
\hline Solar spectral density & $P_{s d}$ & $1367 \mathrm{~W} / \mathrm{m}^{2}$ \\
\hline Solar cell surface area on one spacecraft face & $S$ & $212.8 \mathrm{~cm}^{2}$ \\
\hline
\end{tabular}

